\title{
Plasma fibrinogen and mortality in patients undergoing peritoneal dialysis: a prospective cohort study
}

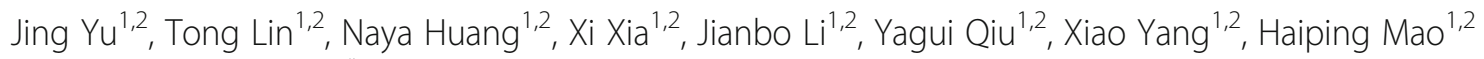
and Fengxian Huang ${ }^{1,2^{*}}$ (1)

\begin{abstract}
Background: Plasma fibrinogen is significantly associated with cardiovascular (CV) events and mortality in the general population. However, the association between plasma fibrinogen and mortality in patients undergoing peritoneal dialysis (PD) is unclear.

Methods: This was a prospective cohort study. A total of 1603 incident PD patients from a single center in South China were followed for a median of 46.7 months. A Cox regression analysis was used to evaluate the independent association of plasma fibrinogen with CV and all-cause mortality. Models were adjusted for age, sex, smoking, a history of CV events, diabetes, body mass index, systolic blood pressure, hemoglobin, blood platelet count, serum potassium, serum albumin, low-density lipoprotein cholesterol, high-density lipoprotein cholesterol, hypersensitive C-reactive protein, estimated glomerular filtration rate, antiplatelet agents and lipid-lowering drugs.

Results: The mean age was $47.4 \pm 15.3$ years, 955 (59.6\%) patients were male, 319 (19.9\%) had a history of CV events, and 410 (25.6\%) had diabetes. The average plasma fibrinogen level was $4.12 \pm 1.38 \mathrm{~g} / \mathrm{L}$. Of the $474(29.6 \%)$ patients who died during follow-up, 235 (49.6\%) died due to CV events. In multivariable models, the adjusted hazard ratios (HRs) for quartile 1, quartile 3, and quartile 4 versus quartile 2 were 1.18 (95\% confidence interval [Cl], $0.72-1.95, P=0.51), 1.47(95 \% \mathrm{Cl}, 0.93-2.33, P=0.10)$, and $1.78(95 \% \mathrm{Cl}, 1.15-2.77, P=0.01)$ for $\mathrm{CV}$ mortality and 1.20 $(95 \% \mathrm{Cl}, 0.86-1.68, P=0.28), 1.29(95 \% \mathrm{Cl}, 0.93-1.78, P=0.13)$, and $1.53(95 \% \mathrm{Cl}, 1.12-2.09, P=0.007)$ for all-cause mortality, respectively. A nonlinear relationship between plasma fibrinogen and CV and all-cause mortality was observed.
\end{abstract}

Conclusions: An elevated plasma fibrinogen level was significantly associated with an increased risk of CV and allcause mortality in patients undergoing PD.

Keywords: Plasma fibrinogen, Peritoneal dialysis, Cardiovascular mortality, All-cause mortality

\footnotetext{
* Correspondence: hfxyl@163.net

${ }^{1}$ Department of Nephrology, The First Affiliated Hospital, Sun Yat-sen University, Guangzhou, Guangdong Province, China

${ }^{2}$ Key Laboratory of Nephrology, National Health Commission and Guangdong Province, The First Affiliated Hospital, Sun Yat-sen University, Guangzhou, Guangdong Province, China
}

(c) The Author(s). 2020 Open Access This article is licensed under a Creative Commons Attribution 4.0 International License, which permits use, sharing, adaptation, distribution and reproduction in any medium or format, as long as you give appropriate credit to the original author(s) and the source, provide a link to the Creative Commons licence, and indicate if changes were made. The images or other third party material in this article are included in the article's Creative Commons licence, unless indicated otherwise in a credit line to the material. If material is not included in the article's Creative Commons licence and your intended use is not permitted by statutory regulation or exceeds the permitted use, you will need to obtain permission directly from the copyright holder. To view a copy of this licence, visit http://creativecommons.org/licenses/by/4.0/ The Creative Commons Public Domain Dedication waiver (http://creativecommons.org/publicdomain/zero/1.0/) applies to the data made available in this article, unless otherwise stated in a credit line to the data. 


\section{Background}

Patients with end-stage renal disease (ESRD) exhibit a high risk for cardiovascular (CV) event morbidity and mortality [1]. The high prevalence of diabetes, hypertension, dyslipidemia, and other traditional CV risk factors only partially explains the high CV risk of these individuals [2]. Some nontraditional CV risk factors associated with the inflammation-coagulation axis, such as hypersensitive C-reactive protein (hs-CRP), hyperhomocysteinemia, and increased prevalence of abnormal altered coagulation with declining renal function, have been reported to be new strong CV risk factors [3-5]. Plasma fibrinogen plays an important role in the coagulation cascade; levels that are too high or too low may cause an increased risk of thrombosis and bleeding, respectively. Studies have shown that the fibrinogen level is influenced by some traditional CV risk factors, such as age, smoking, diabetes, and hypertension, as well as emerging risk factors, such as inflammation [6, 7]. Therefore, fibrinogen may not only represent a risk factor [8] but also provide a common pathway for the interaction of various risk factors promoting CV events.

Fibrinogen is an independent risk factor of CV events and mortality in the general population $[9,10]$. However, it has been reported that uremia has a negative effect on hemostasis, which is called uremic coagulopathy. Uremic coagulopathy causes increased production and/or decreased clearance of procoagulant proteins, leading to increased fibrinogen levels [11-13]. ESRD patients are not a homogeneous cohort, as they include patients undergoing hemodialysis (HD) and peritoneal dialysis (PD). Thus, the coagulation profile of these individuals is not completely the same, which makes the relationship between fibrinogen and mortality complicated and controversial. Elevated plasma fibrinogen independently predicts CV events in individuals with stage 3-4 chronic kidney disease (CKD) [14]. However, the study conducted by Shlipak et al. [15] found that fibrinogen could not predict adverse outcomes. Zoccali et al. [16] found a positive association between high plasma fibrinogen and mortality in HD patients, but this has been contradicted by other studies conducted on HD patients $[17,18]$. Patients with PD can have metabolic abnormalities such as insulin resistance, dyslipidemia, and metabolic syndrome due to longterm exposure to glucose-based dialysate, which links endothelial dysfunction, inflammation, and a tendency toward procoagulation together [19]. Therefore, patients on PD have higher fibrinogen levels and a more prothrombotic profile than patients on HD [20, 21]. We hypothesized that elevated plasma fibrinogen levels are significantly associated with mortality in PD patients. Until now, research on the association between plasma fibrinogen and mortality in PD patients has been limited, and the sample sizes of these studies have been relatively small $[22,23]$. Thus, the aim of this study was to examine the association of plasma fibrinogen with $\mathrm{CV}$ and all-cause mortality in a large cohort of PD patients.

\section{Methods \\ Participants}

From January 1, 2006, to December 31, 2013, we enrolled 1955 patients who initiated PD therapy at a single PD center of the First Affiliated Hospital of Sun Yat-sen University in South China. Eligible patients included those older than 18 years who had undergone catheterization for PD at our center, received PD for more than 3 months, and signed informed consent. We excluded patients who had undergone long-term HD (more than 3 months), those who had received a kidney transplant, those with malignant tumors, those who lacked the baseline fibrinogen data, and those who had an outlier plasma fibrinogen concentration $<0.5$ th or $>$ 99.5th percentile of the observed values. In the end, we included 1603 patients and followed up with them until August 31, 2018.

\section{Data collection and study protocol}

This was a prospective cohort study conducted at our PD center. Baseline demographic data, including age, sex, smoking, a history of $\mathrm{CV}$ events, diabetes, and hypertension, were collected at the initiation of PD therapy. A history of CV events was defined as a patient who had one or more of the following CV events: angina, myocardial infarction, heart failure, angioplasty, coronary artery bypass or stroke. Diabetic patients were those who met the clinical diagnostic criteria for diabetes mellitus and/or those who currently or previously used insulin or oral hypoglycemic agents. Hypertensive patients were those who had at least two separate blood pressure measurements above $140 / 90 \mathrm{mmHg}$ and/or those who used antihypertensive drugs currently or previously.

Clinical and biochemical data, including body mass index (BMI), blood pressure, medication use, plasma fibrinogen, hemoglobin, blood platelet count, serum potassium, serum albumin, serum creatinine, total cholesterol (TC), triglycerides (TG), low-density lipoprotein cholesterol (LDL-C), high-density lipoprotein cholesterol (HDLC), and hs-CRP levels, were collected 3 months after PD therapy initiation. The blood samples were all measured at the same Inspection Center of the First Affiliated Hospital of Sun Yat-sen University. Plasma fibrinogen was measured by a commercial assay reagent (Dade Thrombin Reagent, Siemens, Germany). The normal range of plasma fibrinogen measured in our hospital was $2-4 \mathrm{~g} / \mathrm{L}$. The estimated glomerular filtration rate (eGFR) was used to evaluate baseline residual renal function, which was calculated by the Chronic Kidney Disease Epidemiology Collaboration (CKD-EPI) equation. In the process of $\mathrm{PD}$, the 
dextrose concentration of peritoneal dialysate was usually $1.5 \%$ or $2.5 \%$.

Medication usage data were derived from prescriptions. Antiplatelet agents included aspirin, clopidogrel, and dipyridamole. Lipid-lowering drugs included statins and fibrates. Patients were requested to return to our center quarterly for a comprehensive medical assessment and were interviewed by telephone or face to face monthly by trained nurses and physicians to assess their general condition and adjust the use of medications.

The primary outcome of this study was CV mortality; the secondary outcome was all-cause mortality. CV mortality referred to death from acute myocardial infarction, atherosclerotic heart disease, congestive heart failure, cardiac arrhythmia, sudden death, cardiomyopathy, ischemic or hemorrhagic stroke, and peripheral vascular disease. In the case of in-hospital death, death certificates were used to identify the exact cause of death. In the case of out-ofhospital death, family members were interviewed by telephone to ascertain the circumstances of death. Subsequently, the cause of death was determined by study physicians based on a series of comprehensive considerations of signs and symptoms before and after death, the history and recent health status, and descriptions provided by the patient's family members. All patients were followed up until death, kidney transplantation, transfer to HD therapy, transfer to other centers, loss to follow-up, or the end of follow-up on August 31, 2018. This study was conducted in compliance with the principles of the Declaration of Helsinki and was approved by the Clinical Research Ethics Committee of the First Affiliated Hospital of Sun Yat-sen University. All patients signed informed consent before they entered the study.

\section{Statistical analysis}

The study population was divided into quartiles according to plasma fibrinogen levels: quartile $1,<3.19$; quartile 2, 3.19-3.80; quartile 3, 3.80-4.72; and quartile 4, > 4.72. Quantitative data were tested for normality by the Kolmogorov-Smirnov test, and data were considered normally distributed if the $P$ value was $>0.05$. Data are described as means \pm standard deviations for normally distributed continuous variables, as medians (interquartile ranges, 1/4-3/4) for non-normally distributed continuous variables, and as frequencies (percentages) for categorical data. Comparisons of clinical variables according to plasma fibrinogen quartiles were performed using one-way analysis of variance (ANOVA), the Kruskal-Wallis test, or the chi-squared test according to appropriate corresponding data types. A multiple linear regression was used to explore the independent associated factors of plasma fibrinogen levels, which were adjusted for significant covariates in a simple linear regression using a stepwise conditional method.
Survival was analyzed by the Kaplan-Meier method, and differences in survival distributions among plasma fibrinogen quartiles were evaluated by a Mantel (logrank) test. A multivariable Cox proportional hazards method was used to evaluate the independent association of plasma fibrinogen levels with $\mathrm{CV}$ and all-cause mortality.

First, plasma fibrinogen was divided into quartiles. Given that increased or decreased fibrinogen levels may be associated with $\mathrm{CV}$ and all-cause mortality, we chose the second quartile $(\mathrm{Q} 2)$ as the reference. In the multivariable model, model 1 adjusted for basic covariates (age and sex). Model 2 included model 1 covariates plus comorbid conditions (smoking, a history of $\mathrm{CV}$ events, diabetes, BMI, and systolic blood pressure). Model 3 included model 2 covariates plus biochemical data (hemoglobin, blood platelet count, serum potassium, serum albumin, LDL-C, HDL-C, hs-CRP, and eGFR). Model 4 included model 3 covariates and treatments (antiplatelet agents and lipid-lowering drugs). We used the hazard ratio (HR) and $95 \%$ confidence interval $(\mathrm{CI})$ to describe the results.

Second, we explored the continuous, potentially nonlinear, relationship between plasma fibrinogen and mortality by using fully adjusted restricted cubic spline models. $P<0.05$ was considered statistically significant. Statistical analyses were performed using SPSS software (SPSS, version 13.0, IBM Corp., Chicago, IL, United States) and STATA software (STATA, version 14.0, Stata Corp LP, College Station, TX, United States).

\section{Results}

\section{Participants}

A total of 1955 patients undergoing PD were enrolled at a single PD center. In the end, 1603 incident PD patients were finally included in this study and followed for a median of 46.7 (maximum, 152.5) months (Fig. 1). The baseline characteristics of the study patients are shown in Table 1. Their mean age was $47.4 \pm 15.3$ years, 955 (59.6\%) were male, 319 (19.9\%) had a history of CV events, and $410(25.6 \%)$ had diabetes. The average plasma fibrinogen level was $4.12 \pm 1.38 \mathrm{~g} / \mathrm{L}$. Older age, a higher proportion of smoking, a history of CV events, diabetes, and the use of antiplatelet agents and lipidlowering drugs were prominent in higher quartiles. Moreover, with regard to biochemical data, patients with higher plasma fibrinogen levels had higher blood platelet counts, TC, TG, LDL-C, and hs-CRP levels but lower serum albumin and HDL-C levels.

At the end of the follow-up, 351 (21.9\%) patients continued PD treatment, 368 (23.0\%) underwent kidney transplantation, $273(17.0 \%)$ transferred to HD treatment, 75 (4.7\%) transferred to other centers, and 62 (3.9\%) were lost to follow-up. We recorded 474 (29.6\%) deaths, of which 235 (49.7\%) were due to CV events. 


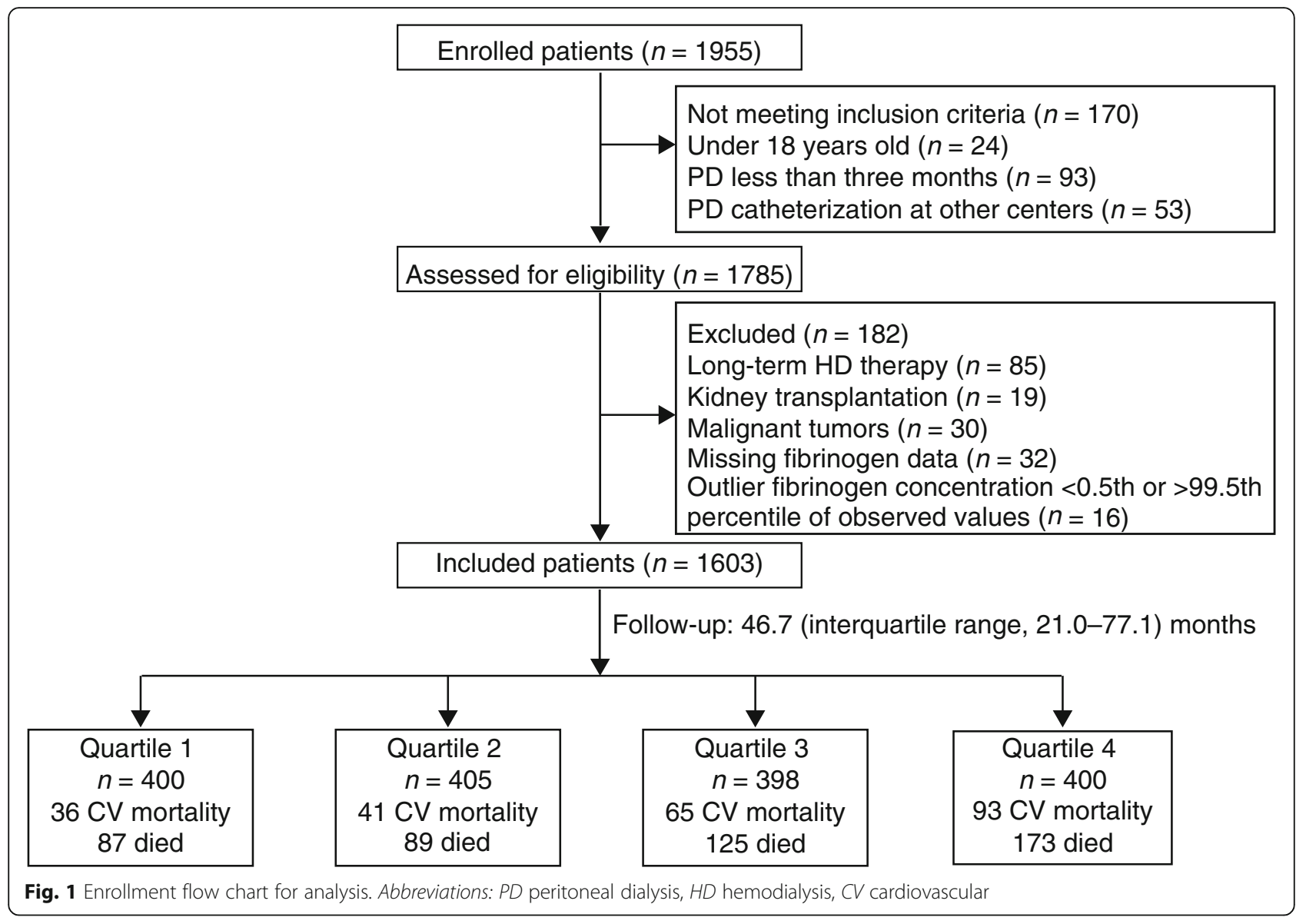

Other causes of death were as follows: 99 (20.9\%), infection; 13 (2.7\%), malignant tumor; 13 (2.7\%), gastrointestinal hemorrhage; 22 (4.6\%), failure/dyscrasia; 11 (2.3\%), giving up treatment; 51 (10.8\%), unknown causes; and 30 (6.3\%), other causes.

\section{Independent factors associated with plasma fibrinogen levels}

Table 2 shows independent associated factors of plasma fibrinogen levels. These values were obtained by adjusting for significant covariates in a simple linear regression (Additional file 1: Table S1) and using a stepwise selection procedure. Plasma fibrinogen levels were independently positively linearly associated with age $(\beta=0.07, P=$ $0.01)$, diabetes $(\beta=0.08, P=0.005)$, BMI $(\beta=0.07, P=$ 0.009), blood platelet count $(\beta=0.24, P<0.001)$, LDL-C $(\beta=0.10, P<0.001)$, and hs-CRP $(\beta=0.19, P<0.001)$, while negatively linearly associated with serum potassium $(\beta=-0.08, P=0.001)$, serum albumin $(\beta=-0.12$, $P<0.001)$, and HDL-C $(\beta=-0.07, P=0.004)$.

\section{Plasma fibrinogen and CV and all-cause mortality}

Kaplan-Meier survival curves of plasma fibrinogen quartiles are shown in Fig. 2. CV and all-cause mortality (Fig. 2a and b, respectively) in patients in quartile 4 were significantly higher than those in quartile 2 (log-rank test $P<0.001$, for both $\mathrm{CV}$ and all-cause mortality).

Table 3 lists the association between plasma fibrinogen and CV and all-cause mortality. In multivariable model 4, the adjusted HRs for quartile 1, quartile 3 , and quartile 4 versus quartile 2 were 1.18 (95\% CI, $0.72-1.95, P=0.51$ ), 1.47 (95\% CI, 0.93-2.33, $P=0.10$ ), and 1.78 (95\% CI, 1.15-2.77, $P=0.01$ ) for CV mortality and 1.20 (95\% CI, 0.86-1.68, $P=0.28$ ), 1.29 (95\% CI, 0.93-1.78, $P=0.13$ ), and 1.53 (95\% CI, 1.12-2.09, $P=0.007)$ for all-cause mortality, respectively.

Additionally, when we examined plasma fibrinogen as a continuous variable in multivariable adjusted cubic spline models, we found that the relationship between fibrinogen and $\mathrm{CV}$ and all-cause mortality was nonlinear, exhibiting approximate J-shaped curves (Fig. 3).

\section{Discussion}

In this study, an elevated plasma fibrinogen level was significantly associated with an increased risk of CV and all-cause mortality in patients undergoing $\mathrm{PD}$, and the relationship between plasma fibrinogen and mortality was nonlinear. 
Table 1 Baseline characteristics of the study cohort according to plasma fibrinogen quartiles

\begin{tabular}{|c|c|c|c|c|c|c|}
\hline \multirow[t]{2}{*}{ Variables } & \multirow{2}{*}{$\begin{array}{l}\text { Total } \\
(n=1603)\end{array}$} & \multicolumn{4}{|c|}{ Plasma fibrinogen quartiles } & \multirow[t]{2}{*}{$P$} \\
\hline & & $\begin{array}{l}\mathrm{Q} 1(<3.19) \\
(n=400)\end{array}$ & $\begin{array}{l}\text { Q2 (3.19-3.80) } \\
(n=405)\end{array}$ & $\begin{array}{l}\text { Q3 (3.80-4.72) } \\
(n=398)\end{array}$ & $\begin{array}{l}\text { Q4 (> 4.72) } \\
(n=400)\end{array}$ & \\
\hline Plasma fibrinogen $(\mathrm{g} / \mathrm{L})$ & $4.12 \pm 1.38$ & $2.72 \pm 0.35$ & $3.48 \pm 0.18$ & $4.26 \pm 0.27$ & $6.04 \pm 1.16$ & $<0.001$ \\
\hline Age (y) & $47.4 \pm 15.3$ & $43.9 \pm 14.9$ & $45.9 \pm 15.6$ & $47.8 \pm 14.5$ & $52.1 \pm 14.8$ & $<0.001$ \\
\hline Male, n (\%) & 955 (59.6) & $220(55.0)$ & $234(57.8)$ & $245(61.6)$ & $256(64.0)$ & 0.05 \\
\hline Smokers, n (\%) & 331 (20.6) & $65(16.3)$ & $76(18.8)$ & $81(20.4)$ & $109(27.3)$ & 0.001 \\
\hline History of CV events, n (\%) & 319 (19.9) & $47(11.8)$ & $61(15.1)$ & $89(22.4)$ & $122(30.5)$ & $<0.001$ \\
\hline Diabetes, n (\%) & $410(25.6)$ & $49(12.3)$ & 79 (19.5) & $114(28.6)$ & $168(42.0)$ & $<0.001$ \\
\hline Hypertension, n (\%) & $1416(88.3)$ & $353(88.3)$ & $358(88.4)$ & $356(89.4)$ & $349(87.3)$ & 0.82 \\
\hline BMI $\left(\mathrm{kg} / \mathrm{m}^{2}\right)$ & $22.04 \pm 3.29$ & $21.15 \pm 2.78$ & $21.66 \pm 3.15$ & $22.31 \pm 3.19$ & $23.07 \pm 3.68$ & $<0.001$ \\
\hline $\mathrm{SBP}(\mathrm{mmHg})$ & $154.3 \pm 24.8$ & $150.4 \pm 24.4$ & $154.8 \pm 23.7$ & $157.3 \pm 27.2$ & $154.8 \pm 23.5$ & 0.002 \\
\hline $\mathrm{DBP}(\mathrm{mmHg})$ & $88.4 \pm 15.6$ & $88.4 \pm 16.3$ & $88.6 \pm 14.8$ & $89.6 \pm 16.1$ & $86.7 \pm 15.3$ & 0.07 \\
\hline Hemoglobin (g/L) & $79.1 \pm 18.6$ & $78.9 \pm 19.1$ & $77.9 \pm 18.9$ & $80.0 \pm 18.6$ & $79.8 \pm 18.0$ & 0.36 \\
\hline Blood platelet count $\left(10 \times 10^{9} / \mathrm{L}\right)$ & $19.3 \pm 8.0$ & $16.5 \pm 6.6$ & $17.7 \pm 7.2$ & $19.8 \pm 7.5$ & $23.4 \pm 8.9$ & $<0.001$ \\
\hline Serum potassium (mmol/L) & $4.65 \pm 0.84$ & $4.69 \pm 0.83$ & $4.73 \pm 0.80$ & $4.68 \pm 0.84$ & $4.50 \pm 0.86$ & $<0.001$ \\
\hline Serum albumin (g/L) & $34.8 \pm 5.1$ & $35.9 \pm 5.1$ & $35.1 \pm 4.7$ & $34.7 \pm 5.0$ & $33.3 \pm 5.3$ & $<0.001$ \\
\hline TC $(\mathrm{mmol} / \mathrm{L})$ & $4.72 \pm 1.43$ & $4.44 \pm 1.31$ & $4.53 \pm 1.13$ & $4.91 \pm 1.60$ & $4.98 \pm 1.57$ & $<0.001$ \\
\hline $\mathrm{TG}(\mathrm{mmol} / \mathrm{L})$ & $1.30(0.93-1.88)$ & $1.15(0.80-1.61)$ & $1.26(0.94-1.84)$ & $1.32(0.97-1.88)$ & $1.49(1.02-2.17)$ & $<0.001$ \\
\hline LDL-C (mmol/L) & $2.83 \pm 1.09$ & $2.59 \pm 0.97$ & $2.69 \pm 0.85$ & $3.00 \pm 1.23$ & $3.05 \pm 1.19$ & $<0.001$ \\
\hline $\mathrm{HDL}-\mathrm{C}(\mathrm{mmol} / \mathrm{L})$ & $1.05 \pm 0.33$ & $1.11 \pm 0.34$ & $1.04 \pm 0.32$ & $1.05 \pm 0.33$ & $1.00 \pm 0.31$ & $<0.001$ \\
\hline $\mathrm{hs}-\mathrm{CRP}$ (mg/L) & $2.23(0.74-8.49)$ & $1.16(0.44-4.08)$ & $1.66(0.49-6.29)$ & $2.91(1.01-9.50)$ & $5.09(1.47-11.79)$ & $<0.001$ \\
\hline eGFR (mL/min/1.73 m²) & $5.2 \pm 2.4$ & $5.3 \pm 2.4$ & $5.1 \pm 2.2$ & $5.2 \pm 2.6$ & $5.3 \pm 2.5$ & 0.62 \\
\hline Antiplatelet agents, n (\%) & $182(11.4)$ & $25(6.3)$ & $38(9.4)$ & $46(11.6)$ & $73(18.3)$ & $<0.001$ \\
\hline Lipid-lowering drugs, n (\%) & $182(11.4)$ & $23(5.8)$ & $40(9.9)$ & $49(12.3)$ & $70(17.5)$ & $<0.001$ \\
\hline
\end{tabular}

Note: Continuous quantitative variables are described as means \pm standard deviations or medians (interquartile ranges, 1/4-3/4). Categorical data are described as frequencies (percentages)

Abbreviations: Q1-Q4 lowest to highest quartile, $C V$ cardiovascular, BMI body mass index, SBP systolic blood pressure, DBP diastolic blood pressure, TC total cholesterol, $T G$ triglycerides, LDL-C low-density lipoprotein cholesterol, HDL-C high-density lipoprotein cholesterol, $h s-C R P$ hypersensitive C-reactive protein, eGFR estimated glomerular filtration rate

We found that the average plasma fibrinogen level in PD patients was higher than the normal range. There are some possible explanations for this phenomenon. First, with the loss of albumin in the peritoneal dialysate, free fatty acids accumulate in the blood, and these are strong stimulating factors for fibrinogen synthesis by the liver $[24,25]$. Second, long-term and continuous exposure to glucose-based dialysate can produce significant metabolic disorders such as hyperinsulinemia, metabolic syndrome, and dyslipidemia, leading to endothelial dysfunction, inflammation, and prothrombotic tendency. Thus, PD patients showed an elevated fibrinogen level and a more prothrombotic profile than HD patients $[20,21]$. In addition, with the decline of renal function, the clearance of fibrinogen and other procoagulant proteins by the kidneys is weakened $[11,12]$.

Our study found that high plasma fibrinogen levels were significantly associated with $\mathrm{CV}$ and all-cause mortality, which is consistent with some studies conducted on stage 3-4 CKD and HD patients [14, 16]. Studies have shown that in CKD patients, the expression level of tissue factor, the key initiating factor of the coagulation cascade, is increased, and the exogenous coagulation pathway is activated [26, 27]. When thrombin is formed, fibrinogen is converted into fibrin, which is mediated by factor XIII to form a cross-linked network around the platelet plug. The size of the fibrin fibers and the density of the overall clot are directly related to the level of fibrinogen [20]. Due to the long-term and continuous exposure to glucose-based dialysate, PD patients are prone to develop advanced glycation end products coupled with chronic inflammation and oxidized stress [11-13, 20]. In this case, fibrinogen becomes glycated and later oxidized through posttranslational modifications $[17,28]$. Some studies have investigated the effect of nonenzymatic glycosylation and the oxidation of fibrinogen on the properties of clots. Nonenzymatic glycosylation and oxidized fibrinogen may reduce clot permeability [29], increase fiber density, or 
Table 2 Independent associated factors of plasma fibrinogen levels in a multiple linear regression model

\begin{tabular}{|c|c|c|c|c|c|}
\hline \multirow[t]{2}{*}{ Variables } & \multicolumn{2}{|c|}{ Unstandardized coefficients } & \multirow{2}{*}{$\begin{array}{l}\text { Standardized coefficients } \\
\beta\end{array}$} & \multirow[t]{2}{*}{$\mathrm{t}$} & \multirow[t]{2}{*}{$P$} \\
\hline & B & Standard error & & & \\
\hline Age (years) & 0.006 & 0.002 & 0.07 & 2.46 & 0.01 \\
\hline Diabetes (yes/no) & 0.25 & 0.09 & 0.08 & 2.79 & 0.005 \\
\hline $\mathrm{BMI}\left(\mathrm{kg} / \mathrm{m}^{2}\right)$ & 0.03 & 0.01 & 0.07 & 2.62 & 0.009 \\
\hline Blood platelet count (per $10 \times 10^{9} / \mathrm{L}$ greater) & 0.04 & 0.004 & 0.24 & 9.31 & $<0.001$ \\
\hline Serum potassium (mmol/L) & -0.13 & 0.04 & -0.08 & -3.35 & 0.001 \\
\hline Serum albumin $(g / L)$ & -0.03 & 0.007 & -0.12 & -4.66 & $<0.001$ \\
\hline LDL-C (mmol/L) & 0.12 & 0.03 & 0.10 & 3.77 & $<0.001$ \\
\hline $\mathrm{HDL}-\mathrm{C}(\mathrm{mmol} / \mathrm{L})$ & -0.30 & 0.10 & -0.07 & -2.89 & 0.004 \\
\hline hs-CRP (per log-unit greater) & 0.18 & 0.02 & 0.19 & 7.32 & $<0.001$ \\
\hline
\end{tabular}

Note: Analysis was performed to explore the independent factors linearly associated with plasma fibrinogen levels in a multiple linear regression model. $F=47.31$, $P<0.001, R^{2}=0.243$, adjusted $R^{2}=0.237$. Covariates for adjustment are listed in Additional file 1: Table S1, stepwise conditional adjustment. Hypersensitive Creactive protein was log-transformed

Abbreviations: BMI body mass index, $L D L-C$ low-density lipoprotein cholesterol, $H D L-C$ high-density lipoprotein cholesterol, $h s-C R P$ hypersensitive $C$-reactive protein

decrease porosity coupled with a decreased individual fiber diameter, leading to an increase in the proportion of thin fibers and the formation of stiffer clots, which are less sensitive to plasmin and more difficult to lyse [30-33]. An increased clot density due to elevated fibrinogen levels has been reported to be independently and strongly associated with CV and all-cause mortality in dialysis patients [17]. This explanation is supported by the fact that in diabetic patients, fibrinogen has been shown to be glycated to form a denser clot and become resistant to fibrinolysis [34-36], and with the improvement of blood glucose control, the glycation of fibrinogen is reduced and the properties of clots are improved, including a decrease in lateral aggregation, an increase in permeability and the rate of fibrinolysis, and a decrease in the proportion of thin fibers in the overall clot [37]. In addition, post-translational modifications of fibrinogen include guanidinylation as well as glycation, which can also change the properties of clots. In particular, it can reduce the diameter of fibers, increase the formation of thin fibers and stiffer clots, and prolong the fibrinolysis time, thereby increasing the risk of thromboembolism morbidity and mortality [17].

Shlipak et al. [15] found that fibrinogen failed to predict adverse outcomes in individuals with CKD. Their conclusions contradict ours. This may be because, first, their cohort was different, and they investigated patients with a broader spectrum of CKD stages, while we focused only on PD patients. Second, in their study, the
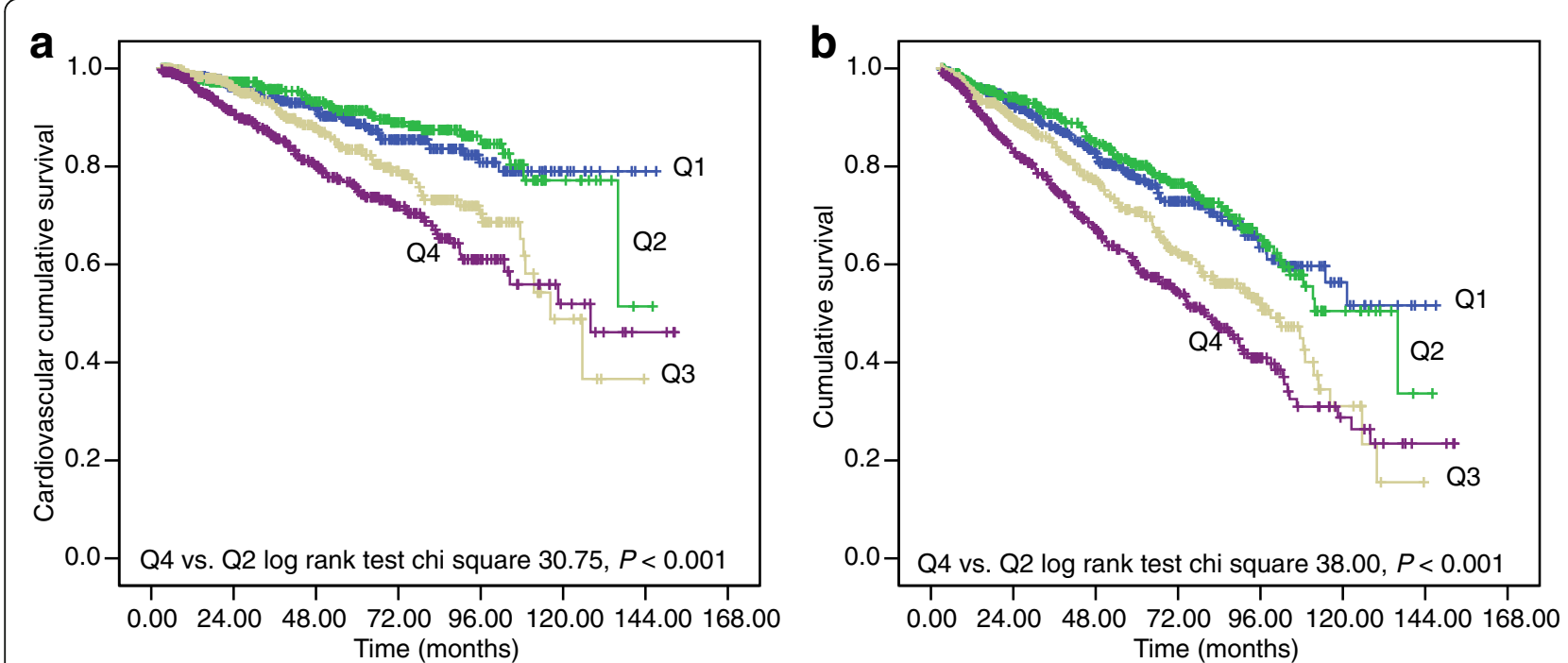

Fig. 2 Cardiovascular and all-cause mortality-free survival curves according to plasma fibrinogen quartiles. a Cardiovascular mortality-free survival curves according to plasma fibrinogen quartiles. b All-cause mortality-free survival curves according to plasma fibrinogen quartiles. Abbreviations: Q1 to Q4 lowest to highest quartile 
Table 3 Association of plasma fibrinogen quartiles with CV and all-cause mortality

\begin{tabular}{|c|c|c|c|c|c|c|}
\hline \multirow[t]{2}{*}{ Variables } & \multicolumn{2}{|l|}{ Q1 $(n=400)$} & \multicolumn{2}{|l|}{ Q3 $(n=398)$} & \multicolumn{2}{|l|}{ Q4 $(n=400)$} \\
\hline & $\mathrm{HR}(95 \% \mathrm{Cl})$ & $P$ & $\mathrm{HR}(95 \% \mathrm{Cl})$ & $P$ & $\mathrm{HR}(95 \% \mathrm{Cl})$ & $P$ \\
\hline \multicolumn{7}{|l|}{ CV mortality } \\
\hline Unadjusted & $0.86(0.55-1.34)$ & 0.49 & $1.74(1.18-2.58)$ & 0.005 & $2.43(1.68-3.51)$ & $<0.001$ \\
\hline Model $1^{\mathrm{a}}$ & $0.92(0.59-1.44)$ & 0.71 & $1.58(1.07-2.34)$ & 0.02 & $1.93(1.33-2.79)$ & $<0.001$ \\
\hline Model $2^{b}$ & $1.04(0.65-1.64)$ & 0.89 & $1.42(0.94-2.13)$ & 0.09 & $1.74(1.19-2.55)$ & 0.004 \\
\hline Model $3^{c}$ & $1.18(0.72-1.94)$ & 0.52 & $1.46(0.92-2.32)$ & 0.11 & $1.76(1.13-2.72)$ & 0.01 \\
\hline Model $4^{d}$ & $1.18(0.72-1.95)$ & 0.51 & $1.47(0.93-2.33)$ & 0.10 & $1.78(1.15-2.77)$ & 0.01 \\
\hline \multicolumn{7}{|c|}{ All-cause mortality } \\
\hline Unadjusted & $0.95(0.71-1.28)$ & 0.74 & $1.54(1.18-2.03)$ & 0.002 & $2.09(1.62-2.70)$ & $<0.001$ \\
\hline Model $1^{\text {a }}$ & $1.00(0.75-1.35)$ & 0.99 & $1.41(1.08-1.86)$ & 0.01 & $1.67(1.29-2.16)$ & $<0.001$ \\
\hline Model $2^{\mathrm{b}}$ & $1.08(0.80-1.47)$ & 0.60 & $1.26(0.95-1.67)$ & 0.11 & $1.45(1.11-1.89)$ & 0.006 \\
\hline Model $3^{c}$ & $1.19(0.85-1.67)$ & 0.30 & $1.28(0.93-1.76)$ & 0.13 & $1.51(1.11-2.06)$ & 0.009 \\
\hline Model $4^{d}$ & $1.20(0.86-1.68)$ & 0.28 & $1.29(0.93-1.78)$ & 0.13 & $1.53(1.12-2.09)$ & 0.007 \\
\hline
\end{tabular}

Note: The second quartile (Q2) was selected as the reference $(n=405)$. Hypersensitive $C$-reactive protein was log-transformed

Abbreviations: $Q 1$ to $Q 4$ lowest to highest quartile, $H R$ hazard ratio, $\mathrm{Cl}$ confidence interval, $\mathrm{CV}$ cardiovascular

${ }^{a}$ Adjusted for age and sex

${ }^{\mathrm{b}}$ Adjusted for model 1 covariates and smoking, a history of cardiovascular events, diabetes, body mass index, and systolic blood pressure

c Adjusted for model 2 covariates and hemoglobin, blood platelet count, serum potassium, serum albumin, low-density lipoprotein cholesterol, high-density lipoprotein cholesterol, hypersensitive C-reactive protein, and estimated glomerular filtration rate levels

${ }^{d}$ Adjusted for model 3 covariates and the use of antiplatelet agents and lipid-lowering drugs

upper quartile was compared with the other lower 3 quartiles; therefore, it is possible to underestimate or obscure the complex U-shaped or J-shaped relationship between fibrinogen and mortality. Low fibrinogen levels can lead to an increased risk of bleeding, which also increases the risk of $\mathrm{CV}$ event morbidity and mortality [38]. Moreover, the relationships between some nontraditional CV risk factors, such as albumin and hemoglobin, and mortality are nonlinear [14]. Therefore, we further examined the relationship between plasma fibrinogen and mortality by using an adjusted restricted cubic spline method, and we discovered that the relationship between fibrinogen and CV and all-cause mortality was nonlinear, exhibiting approximate J-shaped curves. However, we did not find that lower fibrinogen levels were associated with CV and all-cause mortality.
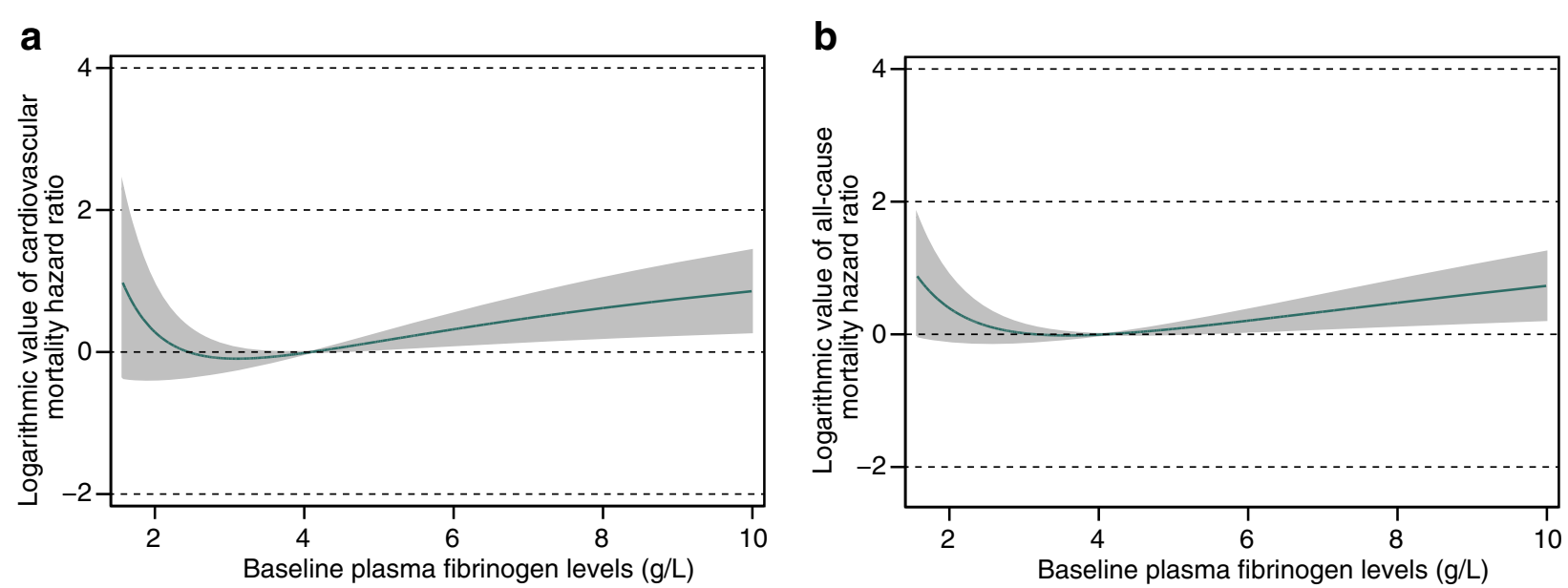

Fig. 3 Nonlinear relationship between fibrinogen and cardiovascular (a) and all-cause (b) mortality. Multivariate adjusted logarithmic value of hazard ratios of cardiovascular and all-cause mortality associated with plasma fibrinogen levels in a Cox regression model using restricted cubic splines, adjusted for age, sex, smoking, a history of cardiovascular events, diabetes, body mass index, systolic blood pressure, hemoglobin, blood platelet count, serum potassium, serum albumin, low-density lipoprotein cholesterol, high-density lipoprotein cholesterol, hypersensitive Creactive protein, estimated glomerular filtration rate, and the use of antiplatelet agents and lipid-lowering drugs. Hypersensitive C-reactive protein was log-transformed 
This is probably because bleeding is multifactorial and can be attributed to thrombocytopenia, uremic disturbance of platelet adhesion and aggregation, morphological changes of the vessels, anemia, coagulation, and fibrinolysis [39-41]. Interactions among multiple factors may mask the effect of the fibrinogen level alone on the prognosis of PD patients.

Our study has several limitations. First, we studied data only at the baseline level and did not consider the impact of data changes on outcomes during the followup period. Second, we did not include platelet function, clot lysis time, $\mathrm{Kt} / \mathrm{V}$, or other factors that may be associated with thrombosis and prognosis, and it is difficult to rule out all residual confounding factors. Third, relying on expert consensus and death certificates to determine the cause of death may lead to misclassification. Finally, all participants came from the Chinese population, limiting its generalization to other ethnic groups.

\section{Conclusions}

In summary, an elevated plasma fibrinogen level was significantly associated with an increased risk of $\mathrm{CV}$ and all-cause mortality in patients undergoing PD, and the relationship between plasma fibrinogen and mortality was nonlinear. These findings provide new insights into the effects of fibrinogen on the prognosis of PD patients. In the future, we need to evaluate whether reducing fibrinogen levels can improve the prognosis of PD patients and clarify the underlying mechanisms.

\section{Supplementary information}

Supplementary information accompanies this paper at https://doi.org/10. 1186/s12882-020-01984-6.

Additional file 1: Table S1. Significant influencing factors for plasma fibrinogen levels in a simple linear regression.

\section{Abbreviations \\ CV: Cardiovascular; PD: Peritoneal dialysis; HR: Hazard ratio; Cl: Confidence interval; ESRD: End-stage renal disease; hs-CRP: Hypersensitive C-reactive pro- tein; HD: Hemodialysis; CKD: Chronic kidney disease; BMI: Body mass index; TC: Total cholesterol; TG: Triglycerides; LDL-C: Low-density lipoprotein cholesterol; HDL-C: High-density lipoprotein cholesterol; eGFR: Estimated glomerular filtration rate; CKD-EPI: Chronic Kidney Disease Epidemiology Collaboration; ANOVA: One-way analysis of variance; SBP: Systolic blood pressure; DBP: Diastolic blood pressure}

\section{Acknowledgements}

The authors appreciate all the nephrologists and nurses in their PD center for their management of PD patients and for providing useful suggestions. The authors also appreciate all PD patients who participated in the study for providing valuable clinical data.

\section{Authors' contributions}

All authors take responsibility for the integrity of the data and the accuracy of data analysis, read and approve the final manuscript. Research idea and study design: JY, NH, and $F H$; data acquisition: $J Y, T L, X X$, JL, and YQ; data analysis/interpretation: JY, TL, NH, and XX; statistical analysis: JY, $T L, N H$, and $J L ;$ article drafting: JY; critical article revision: $F H, X Y$, and $H M$.

\section{Funding}

The National Natural Science Foundation of China (grant 81570750 and 81870575), National Natural Science Foundation for Young Scholars of China (grant 81700718), Natural Science Foundation of Guangdong Province (grants 2017A030310044, 2017A030310199, and 2019A1515010992), National Key R\&D Program of China (grant 2016YFC0906101), Operational Grant of Guangdong Provincial Key Laboratory (grant 2017B030314019), Science and Technology Program of Guangdong Province (grant 2017A050503003), and Guangzhou Municipal Program of Science and Technology (grant

201704020167) all supplied the funds for the establishment of peritoneal dialysis database, collection of follow-up data and detection of blood. The funders played no role in study design, data collection, analysis, interpretation or manuscript writing.

\section{Availability of data and materials}

The datasets used and/or analyzed during the current study are available from the corresponding author on reasonable request.

\section{Ethics approval and consent to participate}

The study was conducted in compliance with the principles of the Declaration of Helsinki and was approved by the Clinical Research Ethics Committee of the First Affiliated Hospital of Sun Yat-sen University. All patients provided their written informed consent before they were enrolled in the study.

\section{Consent for publication}

Not applicable.

\section{Competing interests}

The authors declare that they have no competing interests.

Received: 29 September 2019 Accepted: 27 July 2020

Published online: 17 August 2020

\section{References}

1. Cheng $X$, Nayyar S, Wang M, Li X, Sun Y, Huang W, et al. Mortality rates among prevalent hemodialysis patients in Beijing: a comparison with USRDS data. Nephrol Dial Transplant. 2013;28(3):724-32.

2. Zoccali C. Cardiovascular risk in uraemic patients-is it fully explained by classical risk factors? Nephrol Dial Transplant. 2000;15(4):454-7.

3. Oksa A. Cardiovascular risk in patients with chronic kidney diseases: a time for new risk markers? Bratisl Lek Listy. 2006;107(8):314-9.

4. Parekh RS, Plantinga LC, Kao WHL, Meoni LA, Jaar BG, Fink NE, et al. The association of sudden cardiac death with inflammation and other traditional risk factors. Kidney Int. 2008;74(10):1335-42.

5. Appel LJ. Beyond (or back to) traditional risk factors: preventing cardiovascular disease in patients with chronic kidney disease. Ann Intern Med. 2004;140(1):60-1.

6. Maple-Brown $\sqcup$, Cunningham J, Nandi N, Hodge A, O'Dea K. Fibrinogen and associated risk factors in a high-risk population: urban indigenous Australians, the DRUID study. Cardiovasc Diabetol. 2010;9:69.

7. Levi M, van der Poll T. Inflammation and coagulation. Crit Care Med. 2010; 38(2 Suppl):S26-34.

8. Kannel WB. Influence of fibrinogen on cardiovascular disease. Drugs. 1997; 54(Suppl 3):32-40.

9. Emerging Risk Factors Collaboration, Kaptoge S, Di Angelantonio E, Lowe G, Pepys MB, Thompson SG, et al. C-reactive protein concentration and risk of coronary heart disease, stroke, and mortality: an individual participant metaanalysis. Lancet. 2010;375(9709):132-40.

10. Danesh J, Lewington S, Thompson SG, Lowe GD, Collins R, Kostis JB, et al. Plasma fibrinogen level and the risk of major cardiovascular diseases and nonvascular mortality: an individual participant meta-analysis. JAMA. 2005; 294(14):1799-809.

11. Schlieper G, Hess K, Floege J, Marx N. The vulnerable patient with chronic kidney disease. Nephrol Dial Transplant. 2016;31(3):382-90.

12. Roberts MA, Hare DL, Ratnaike $\mathrm{S}$, lerino FL. Cardiovascular biomarkers in CKD: pathophysiology and implications for clinical management of cardiac disease. Am J Kidney Dis. 2006;48(3):341-60.

13. Shlipak MG, Fried LF, Crump C, Bleyer AJ, Manolio TA, Tracy RP, et al. Elevations of inflammatory and procoagulant biomarkers in elderly persons with renal insufficiency. Circulation. 2003;107(1):87-92. 
14. Weiner DE, Tighiouart H, Elsayed EF, Griffith JL, Salem DN, Levey AS, et al. The relationship between nontraditional risk factors and outcomes in individuals with stage 3 to 4 CKD. Am J Kidney Dis. 2008;51(2):212-23.

15. Shlipak MG, Fried LF, Cushman M, Manolio TA, Peterson D, Stehman-Breen C, et al. Cardiovascular mortality risk in chronic kidney disease: comparison of traditional and novel risk factors. JAMA. 2005;293(14):1737-45.

16. Zoccali C, Mallamaci F, Tripepi G, Cutrupi S, Parlongo S, Malatino LS, et al. Fibrinogen, mortality and incident cardiovascular complications in endstage renal failure. J Intern Med. 2003;254(2):132-9.

17. Schuett K, Savvaidis A, Maxeiner S, Lysaja K, Jankowski V, Schirmer SH, et al. Clot structure: a potent mortality risk factor in patients on hemodialysis. J Am Soc Nephrol. 2017;28(5):1622-30.

18. Selim G, Stojceva-Taneva O, Ivanovski N, Zafirovska K, Sikole A, Trajcevska L, et al. Inflammation and anaemia as predictors of cardiovascular mortality in hemodialysis patients. Hippokratia. 2007;11(1):39-43.

19. Kobayashi M, Yorioka N, Yamakido M. Hypercoagulability and secondary hyperfibrinolysis may be related to abnormal lipid metabolism in patients treated with continuous ambulatory peritoneal dialysis. Nephron. 1997;76(1): 56-61.

20. Brophy DF, Carl DE, Mohammed BM, Song J, Martin EJ, Bostic JL, et al. Differences in coagulation between hemodialysis and peritoneal dialysis. Perit Dial Int. 2014;34(1):33-40.

21. Gäckler A, Rohn H, Lisman T, Benkö T, Witzke O, Kribben A, et al. Evaluation of hemostasis in patients with end-stage renal disease. PLoS One. 2019; 14(2):e0212237.

22. Tekin IO, Pocan B, Borazan A, Ucar E, Kuvandik G, llikhan S, et al. Positive correlation of CRP and fibrinogen levels as cardiovascular risk factors in early stage of continuous ambulatory peritoneal dialysis patients. Ren Fail. 2008; 30(2):219-25.

23. Jovanovic DB, Stosović MD, Gojakovic BM, Jovanovic NZ, Stanojevic-Stosovic ML, Simic-Ogrizovic S P, et al. Inflammatory markers as mortality predictors in continuous ambulatory peritoneal dialysis patients. Ren Fail. 2015;37(2): 230-6.

24. Pickart LR, Thaler MM. Free fatty acids and albumin as mediators of thrombin-stimulated fibrinogen synthesis. Am J Phys. 1976;230(4):996-1002.

25. Prinsen BHCMT, Rabelink TJ, Beutler JJ, Kaysen GA, De Boer J, Boer WH, et al. Increased albumin and fibrinogen synthesis rate in patients with chronic renal failure. Kidney Int. 2003;64(4):1495-504.

26. Adams RLC, Bird RJ. Review article: coagulation cascade and therapeutics update: relevance to nephrology. Part 1: overview of coagulation, thrombophilias and history of anticoagulants. Nephrology. 2009;14(5):462-70.

27. Pawlak K, Tankiewicz J, Mysliwiec M, Pawlak D. Tissue factor/its pathway inhibitor system and kynurenines in chronic kidney disease patients on conservative treatment. Blood Coagul Fibrinolysis. 2009;20(7):590-4.

28. Martinez M, Weisel JW, Ischiropoulos H. Functional impact of oxidative posttranslational modifications on fibrinogen and fibrin clots. Free Radic Biol Med. 2013;65:411-8.

29. Undas A, Nycz K, Pastuszczak M, Stompor T, Zmudka K. The effect of chronic kidney disease on fibrin clot properties in patients with acute coronary syndrome. Blood Coagul Fibrinolysis. 2010;21(6):522-7.

30. Collet JP, Allali Y, Lesty C, Tanguy ML, Silvain J, Ankri A, et al. Altered fibrin architecture is associated with hypofibrinolysis and premature coronary atherothrombosis. Arterioscler Thromb Vasc Biol. 2006:26(11):2567-73.

31. Collet JP, Park D, Lesty C, Soria J, Soria C, Montalescot G, et al. Influence of fibrin network conformation and fibrin fiber diameter on fibrinolysis speed: dynamic and structural approaches by confocal microscopy. Arterioscler Thromb Vasc Biol. 2000;20(5):1354-61.

32. Parastatidis I, Thomson L, Burke A, Chernysh I, Nagaswami C, Visser J, et al. Fibrinogen beta-chain tyrosine nitration is a prothrombotic risk factor. J Biol Chem. 2008;283(49):33846-53.

33. Brownlee M, Vlassara $H$, Cerami A. Nonenzymatic glycosylation reduces the susceptibility of fibrin to degradation by plasmin. Diabetes. 1983;32(7):680-4.

34. Dunn EJ, Philippou H, Ariëns RAS, Grant PJ. Molecular mechanisms involved in the resistance of fibrin to clot lysis by plasmin in subjects with type 2 diabetes mellitus. Diabetologia. 2006;49(5):1071-80.

35. Alzahrani SH, Ajjan RA. Coagulation and fibrinolysis in diabetes. Diab Vasc Dis Res. 2010;7(4):260-73.

36. Dunn EJ, Ariëns RAS, Grant PJ. The influence of type 2 diabetes on fibrin structure and function. Diabetologia. 2005;48(6):1198-206.

37. Pieters M, Covic N, van der Westhuizen FH, Nagaswami C, Baras Y, Toit Loots D, et al. Glycaemic control improves fibrin network characteristics in type 2 diabetes - a purified fibrinogen model. Thromb Haemost. 2008;99(4): 691-700.

38. Tanhehco YC, Berns JS. Red blood cell transfusion risks in patients with endstage renal disease. Semin Dial. 2012;25(5):539-44.

39. Lutz J, Menke J, Sollinger D, Schinzel H, Thürmel K. Haemostasis in chronic kidney disease. Nephrol Dial Transplant. 2014;29(1):29-40.

40. Robinson TN, Wu DS, Sauaia A, Dunn CL, Stevens-Lapsley JE, Moss M, et al. Slower walking speed forecasts increased postoperative morbidity and 1year mortality across surgical specialties. Ann Surg. 2013;258(4):582-90.

41. Mohapatra A, Valson AT, Gopal B, Singh S, Nair SC, Viswabandya A, et al. Hemostatic abnormalities in severe renal failure: do they bark or bite? Indian J Nephrol. 2018;28(2):135-42

\section{Publisher's Note}

Springer Nature remains neutral with regard to jurisdictional claims in published maps and institutional affiliations.
Ready to submit your research? Choose BMC and benefit from:

- fast, convenient online submission

- thorough peer review by experienced researchers in your field

- rapid publication on acceptance

- support for research data, including large and complex data types

- gold Open Access which fosters wider collaboration and increased citations

- maximum visibility for your research: over $100 \mathrm{M}$ website views per year

At BMC, research is always in progress.

Learn more biomedcentral.com/submissions 